\title{
The New Generation of Ionization and Recombination Fronts
}

\author{
J.E. Dyson \& T.W. Hartquist \\ Department of Physics and Astronomy, University of Leeds, Leeds LS2 \\ 9JT, UK
}

\author{
R.J.R. Williams \\ Department of Physics and Astronomy, University of Cardiff, PO Box \\ 913, Cardiff CR24 3YB, UK
}

\author{
M.P. Redman \\ Department of Physics and Astronomy, University College London, \\ London WC1E 6BT, UK
}

\begin{abstract}
We summarize previous work on hydrodynamic ionization fronts and new work where magnetic fields are incorporated into their structures. We describe recent work on recombination front structures and outline refinements which need to be made to both them and ionization fronts.
\end{abstract}

\section{Introduction}

Expanding $\mathrm{H}$ il regions are bounded by ionization fronts (IF). IFs divide into Rtypes, which move supersonically into upstream neutral gas, and D-types, which move subsonically (Kahn 1954). At the edges of the velocity regions allowed by the jump conditions, the ionized flow out of these 'critical' IFs takes place at the isothermal ionized sound speed. IFs in which the gas both enters and leaves the front supersonically are called weak R-type, while in weak D-types the gas enters and leaves subsonically. Strong R-type IFs, in which the gas would experience a supersonic-subsonic transition, are overdetermined and do not exist. In strong D-type IFs, overheating allows a subsonic-supersonic transition within the IF.

Sudden ionization produces an initially weak R-type IF. Once its velocity drops to about twice the sound speed in the ionized gas, a shock moves out of the IF, which becomes weak D-type (Axford, 1961; Goldsworthy 1961; Mathews, 1965; Lasker 1966a). This sequence is relevant when the ionized gas is confined to a region bounded by an IF. The IF incident on an isolated neutral clump can stall and the ionized gas flows from its surface as a wind. The IF is strong D-type and the gas accelerates through the isothermal sonic point inside the IF.

Axford's (1961) study solved the internal structure equations using a simple cooling function and gave the full range of hydrodynamic structures and the evolution from weak $\mathrm{R}$ through strong $\mathrm{D}$ - and weak D-types. He showed that in strong-D IFs, the specific enthalpy of the gas in the front reached a maximum at 
the adiabatic sound speed. Most other steady state studies concentrate on the $\mathrm{D}$ and weak $\mathrm{R}$ solutions. Various authors included numerous ions, molecular hydrogen and radiative transfer (Hjellming 1966; Mendis 1969; Briscoe 1972; Harrington 1977; Hill 1977; Mason 1980; Bertoldi 1989).

The stability and evolution of IFs are better addressed by the calculation of time-dependent global flows. Early calculations were by Mathews (1965) and Lasker (1966a), while Yorke (1986) reviews subsequent work. The finite spatial and temporal dynamic range of these calculations can lead to artificial features within the IF structures themselves but the global evolution of the regions is generally still well described (cf. Bedijn \& Tenorio-Tagle 1984).

There have been many significant recent developments in the study of IFs. Strong-D type IFs around clumps have been directly observed at the surface of photoionized clumps and disks in $\mathrm{H}$ II regions such as Orion (e.g. Henney \& O'Dell 1999), and planetary nebulae (e.g. Dyson et al. 1993), and are found to be of great importance in the structure of ultra-compact $\mathrm{H}$ II regions (e.g. Dyson et al. 1995). Sysoev (1997) found long-wavelength instabilities to occur in Dtype IFs, while Williams (1999) described a shadowing instability of R-type IFs. Franco et al. (2000) review the most recent studies of global structures.

\section{MHD Ionization Fronts}

All diffuse astronomical sources are clumpy (e.g. Hartquist \& Dyson 1993). To date, studies of clump photoevaporation (e.g. Dyson 1968; Bertoldi 1989; Arthur \& Lizano 1997) have treated non-magnetized clumps. However, evidence (e.g. Myers \& Khersonsky 1995) suggests that the ratio of magnetic pressure to thermal pressure in diffuse clouds may be up to ten or so, and even higher in the CO emitting clumps in the Rosette Molecular Cloud.

Lasker (1966b) first studied the jump conditions across MHD IFs. Redman et al. (1998) extended this work to regions of much higher magnetic-to-thermal pressure ratios $(\xi)$. The fast mode sound speed plays the role of the ordinary sound speed in the hydrodynamic case. Importantly, the forbidden velocity gap decreases as $\xi$ increases. D-critical IFs are affected significantly if $\xi \gtrsim 1$. R-critical IFs are affected only at relatively high $\xi(\gtrsim 50)$ (Redman et al. 1998).

Across obliquely magnetized IFs, there are $R$ and D-type IFs about both the fast- and the slow-mode speeds (Williams et al. 2000). If $v_{z, \circ}$ and $v_{z, \mathrm{i}}$ are the gas velocity components perpendicular to the IF in the neutral and ionized gas respectively, $v_{\mathrm{f}}$ and $v_{\mathrm{s}}$ as the fast and slow mode velocities respectively and $v_{\mathrm{A}}$ the Alfven velocity, the possible IFs become fast R-type $\left(v_{z, 0}, v_{z, \mathrm{i}}>v_{\mathrm{f}}\right)$; slow R-type $\left(v_{\mathrm{A}}>v_{z, \mathrm{i}}, v_{z, \mathrm{o}}>v_{\mathrm{s}}\right)$; fast D-type $\left(v_{\mathrm{f}}>v_{z, \mathrm{i}}, v_{z, \mathrm{o}}>v_{\mathrm{A}}\right)$; slow D-type $\left(v_{\mathrm{s}}>v_{z, 0}, v_{z, \mathrm{i}}\right)$. R-type IFs are compressive while D-type IFs are rarefying, as in the hydrodynamic case.

In obliquely-magnetized IFs, we specify two parameters in the upstream neutral gas, the ratios $\xi=B_{x}^{2} / 8 \pi \rho c^{2}$ and $\eta=B_{z}^{2} / 8 \pi \rho c^{2} . B_{x}$ and $B_{z}$ are the magnetic field strength components respectively parallel to and perpendicular to the plane of the IF, $\rho$ is the gas density and $c$ is the isothermal sound speed.

Slow D-critical velocities are strongly affected for even small values of $\xi$ and $\eta$. High enough transverse magnetic fields allow fast-D and slow-R IFs with velocities forbidden to hydrodynamic IFs. Since fast mode shocks increase the 
transverse field, the transition from a fast $\mathrm{R}$-type front to a fast $\mathrm{D}$-type front can take place by the generation of a fast shock ahead of the IF, analogous to the transition from a weak R-type to a weak D-type by the generation of a shock in the hydrodynamic case. Slow mode shocks switch off the transverse magnetic field and allow a transition sequence such as fast $D$ to slow $R$ to slow $D$. The interpretation of magnetic field strengths associated with expanding $\mathrm{H}$ II regions (e.g. Roberts, Crutcher \& Troland 1995) requires considerable caution (Williams et al. 2000).

The internal structures of MHD IFs are complex. Williams \& Dyson (in preparation) have generated the internal structures of a wide variety of MHD IFs using Axford's (1961) approximations.

\section{Recombination Fronts}

Ionized gas recombines if the ionization source is removed (e.g. gas cooling behind supernova driven shock waves). Radiatively ionized regions recombine if the ionizing source is sufficiently dimmed. Generally, the recombining regions are extended but there are astrophysically interesting situations where there is a relatively sharp ionized-neutral interface, a recombination front ( $R F)$, across which there is a steady flux of ionized gas. The velocity of the ionized gas through the RF defines the interface velocity. The thicknesses of RFs are determined by the recombination of ionized gas advected through them and can be broader than IFs (although their thicknesses are comparable to the thermal relaxation distance behind an IF, for similar densities of ionized gas).

RFs are important in, e.g., accretion flows onto stars with strong UV radiation fields (Mestel 1953), ultra-compact $\mathrm{H}$ II regions (Dyson et al. 1995 et seq; Lizano et al. 1996) and recombination zones in mass-loaded jets (Redman \& Dyson 1999).

Newman \& Axford (1968) examined the properties of RFs and found there existed the analogies of the weak $R$, strong $R$ and weak D IFs but there was no analogy to strong D IFs. Williams \& Dyson (1996) re-examined this work and found an additional small, but important, class of transonic solutions. They also found extra supersonic-subsonic solutions generated partly by the insertion of shocks in transonic solutions.

Transonic solutions become important when initially subsonic global flows approach the sonic point as a result of geometrical divergence and/or mass loading, (e.g. if gas 'leaks' through the surface of an $\mathrm{H}$ II region). Exiting supersonic flows can match up to a range of external pressures simply by the insertion of a shock at some radius. Williams (unpublished) has calculated the structures of a series of mass-loaded flows which start subsonically and emerge supersonically through an RF.

\section{Conclusions}

We have described our recent work on the structure of MHD IFs and on RFs. Much more realistic models are still required: to accurately discriminate between MHD and purely hydrodynamic IFs; to determine the effects of magnetic fields on global models of $\mathrm{H}$ II regions and photoionized clumps; to study the effects of 
clumpy media and the transition from IFs to RFs as a result of mass injection. A subject area which seemed to be reasonably well understood forty years ago has become dynamically active again.

\section{References}

Arthur, S.J., Lizano, S. 1997, ApJ, 484

Axford, W.I. 1961, Phil. Trans. R. Soc. Lon. A, 253, 301

Bedijn, P.J., Tenorio-Tagle, C. 1984, A\&A, 135, 81

Bertoldi, F. 1989, ApJ, 346, 735

Briscoe, F. 1972, unpublished thesis, University of Leeds, UK

Dyson, J.E. 1968, Ap\&SS, 1, 388

Dyson, J.E., Hartquist, T.W., Biro, S. 1993, MNRAS, 261, 430

Dyson, J.E., Williams, R.J.R., Redman, M.P. 1995, MNRAS, 277, 700

Franco, J., Kurtz, S.E., Garcia-Segura, G., Hofner, P. 2000, Ap\&SS, 272, 169

Goldsworthy, F.A. 1961, Phil. Trans. R. Soc. Lond. A, 253, 277

Harrington, J.P. 1977, MNRAS, 179, 63

Hartquist, T.W., Dyson, J.E. 1993, Quart. J. R. Astr. Soc., 34, 57

Henney, W.J., O’Dell, C.R. 1999, AJ, 118, 235

Hill, J.K. 1977, ApJ, 212, 685

Hjellming, R.M. 1966, ApJ, 143, 420

Kahn, F.D. 1954, Bull. Astr. Inst. Netherlands, 12, 187

Lasker, B.M. 1966a, ApJ, 143, 700

Lasker, B.M. 1966b, ApJ, 146, 471

Lizano, S, Canto, J., Garay, G., Hollenbach, D. 1996, ApJ, 468, 739

Mason, D.J. 1980, A\&A, 92, 117

Mathews, W.G. 1965, ApJ, 142, 1120

Mendis, D.A. 1969, MNRAS, 142, 241

Mestel, L. 1953, MNRAS, 114, 437

Myers, P.C., Khersonsky, V.K. 1995, ApJ, 442, 186

Newman, R.C., Axford, W.I. 1968, ApJ, 151, 1145

Redman, M.P., Dyson, J.E. 1999, MNRAS, 302, L17

Redman, M.P., Williams, R.J.R., Dyson, J.E., Hartquist, T.W., Fernandez B.R. 1998, A\&A, 331, 1099

Roberts, D.A., Crutcher, R.M., Troland, T.H. 1995, ApJ, 442, 208

Sysoev, N.E. 1997, Astron. Lett., 23, 409

Williams, R.J.R. 1999, MNRAS, 310, 789

Williams, R.J.R., Dyson, J.E. 1996, MNRAS, 279, 987

Williams, R.J.R., Dyson, J.E., Hartquist, T.W. 2000, MNRAS, 314, 315

Yorke, H.W. 1986, ARAA, 24, 246 